\title{
Tyrosine Rotamer States in Beta Amyloid: Signatures of Aggregation and Fibrillation
}

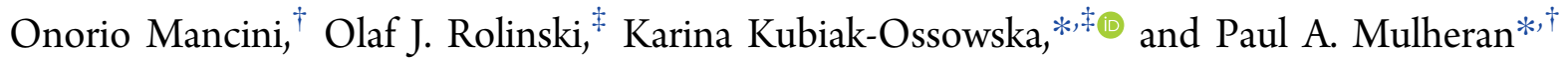 \\ ${ }^{\dagger}$ Department of Chemical and Process Engineering, University of Strathclyde, Glasgow G1 1XJ, U.K. \\ ${ }^{\ddagger}$ Department of Physics, University of Strathclyde, Glasgow G4 0NG, U.K.
}

\section{Supporting Information}

ABSTRACT: During the early stages of $\beta$ amyloid (Ab) peptide aggregation, toxic oligomers form which have been recognized as a likely cause of Alzheimer's disease. In this work, we use fully atomistic molecular dynamics simulation to study the amorphous aggregation of the peptide as well as model $\beta$-sheet protofibril structures. In particular, we study the rotamer states of the single fluorescent tyrosine (Tyr) residue present in each $\mathrm{Ab}$. We find that the occupation of the four previously identified rotamers is different for monomeric and amorphous aggregates because of the differing environments of the Tyr side-chains. Surprisingly, we also identify two new rotamers that uniquely appear for the $\beta$ sheet structures, so that together the rotamers provide distinct signatures for the different stages of aggregation and fibrillation. We propose that these rotamers could be identified in fluorescence spectroscopy, with each rotamer having a distinct fluorescence lifetime because of its different exposures to the solvent. The identification of the two new rotamers therefore provides a new means to probe amyloid formation kinetics and to monitor the effect of additives including prospective drugs.

\section{INTRODUCTION}

Because of advances in the medical field, the average life expectancy of the population of many countries is increasing every year and, consequently, the global population affected by amyloid-related diseases has also been increasing. ${ }^{1,2}$ Amyloidrelated diseases occur when certain proteins, naturally found in the body, stop functioning correctly and begin to rapidly aggregate forming large $\beta$-sheet plaques. During the aggregation phase, extreme cellular degeneration occurs, most likely at the oligomer stage of aggregation, ${ }^{3}$ leading to diseases such as Alzheimer's, type-2 diabetes and Lewy body myositis. The malfunctioning proteins appear to differ for each disease. ${ }^{1,4,5}$ These proteins all form $\beta$-sheet fibrils; in the case of Alzheimer's, the fibrils are deposited in the brain and in type- 2 diabetes, they are deposited in the pancreas. ${ }^{4}$ This paper focuses on the $\beta$ amyloid ( $\mathrm{Ab}$ ) peptide that is associated with Alzheimer's disease.

The natural function of $\mathrm{Ab}$ peptide is not fully understood, and there is no apparent change to (or loss of) physiological functions in animals that have the $\mathrm{Ab}$ peptide missing. ${ }^{6-8}$ However, some potential explanations for the role of $\mathrm{Ab}$ in vivo have been proposed by various researchers; Bogoyevitch et al. ${ }^{9}$ showed that it could be a requirement for kinase enzyme activation, and this has been supplemented by further work in the area. ${ }^{10}$ It may also be related to oxidative stress protection, ${ }^{11,12}$ cholesterol transport regulation, ${ }^{13,14}$ the prevention of microbial activity, ${ }^{15}$ or play a role as a transcription factor. ${ }^{16,17}$ Regardless, the Ab peptides eventually stop functioning correctly and begin to aggregate, creating the toxic oligomer stage that contributes to the disease development.

Despite the significant interest in the research community, there are many aspects of the aggregation process that require better understanding to inform strategies to prevent or modify the aggregation. Experimentally, in vitro techniques can be used to study the aggregation; however, it is difficult to determine the fibril nucleation events that occur on the molecular level. This makes obtaining a detailed understanding of aggregation pathways impossible using one method alone.

$\mathrm{Ab}$ is a small protein (or peptide, with $36-43$ amino acids) that contains a single tyrosine (Tyr) residue, ${ }^{18}$ which allows fluorescence measurements to be used to monitor $\mathrm{Ab}$ aggregation in vitro. The change in fluorescence decays over time observed in these experiments is due to the environmental changes of the Tyr residues and so can be used to infer the temporal progression of the aggregation. ${ }^{19}$ The literature shows that starting with dispersed monomeric $\mathrm{Ab}$ peptide at high concentration, there is a clear lag phase where no aggregate growth occurs, followed by a rapid formation of long mature fibrils containing a backbone held by hydrogen bonding in the form of $\beta$-sheets. Previous research using fluorescence spectroscopy also shows that the Tyr side-chain goes through rapid changes during the initial stages of aggregation, but very quickly stabilises. ${ }^{20}$ This is then followed by the rapid growth phase where fluorescence dye spectros-

Received: September 17, 2018

Accepted: November 8, 2018

Published: November 27, 2018 
copy reveals the rapid formation of $\beta$-sheets. ${ }^{21}$ Once aggregation occurs, the lifetimes associated with the $\mathrm{Tyr}$ side-chains also change, reflecting the new Tyr environments (named rotamer states) not found in the monomer form of $\mathrm{Ab}$.

Though the changes to the fluorescence responses can be measured, understanding how and why these changes occur is not clear through experimentation alone. The work in this paper describes fully atomistic molecular dynamic (MD) simulations, where the primary focus is to observe the effects of differing local environments on the Tyr residues and their resultant conformations. In the previous work, we have shown how MD simulation can help interpret fluorescence anisotropy experiments monitoring $\mathrm{Ab}$ aggregation. ${ }^{22}$ In this study, we reveal new rotamer states that seem to be uniquely associated with fibril structures and absent from monomer or amorphous aggregates. These new rotamers are expected to have their own fluorescence lifetimes because each rotamer exposes the fluorescent side-chains to solvent in a different way. Therefore, our results offer the potential for a new fluorescence spectroscopy feature that can directly monitor fibril nucleation, as opposed to amorphous aggregation, without the need for external labeling with fluorescent dyes. A capability to monitor the formation pathway of the fibrils (monomers to oligomer to fibril) in vitro, and potentially also in vivo, would not only provide new scientific insight but also help in the search for inhibitors to prevent aggregation or for incipients to promote fibril formation from the toxic oligomers.

\section{MATERIALS AND METHODS}

Fully atomistic $\mathrm{MD}$ simulations of $\mathrm{Ab}_{1-42}$ systems were performed in this work. All simulations were performed using NAMD $2.8^{23}$ and the Charmm27 force field, with $\mathrm{VMD}^{24}$ employed to prepare the simulations, visualize the results, and track the orientation of the tyrosine residues. Standard Charmm 27 protonation states have been used, and stability tests for single $\mathrm{Ab}_{1-42}$ peptides in a $\mathrm{NaCl}$ solution show no tendency for abnormal folding; additional data are provided in the Supporting Information. The first set of simulations used monomers and amorphous aggregates, which were created using an NMR structure of $\mathrm{Ab}_{1-42}$ (1iyt.pdb) taken from the protein data bank and obtained by Crescenzi et al. $^{25}$ These systems were compared to potential protofibril structures found by Wälti et al. (2nao.pdb), ${ }^{26}$ using solvent (water) with both zero and physiologically relevant bulk iconicity.

The liyt.pdb structure was copied as required for the various aggregation simulations, and the $\mathrm{Ab}_{1-42}$ peptides were positioned with an adequate distance between them to ensure they aggregated in a natural manner during the simulations. Generally, there was at least $12 \AA$ between peptides at the start of the simulations, except when trying to promote aggregation; in the small water-box three-peptide system (which despite this, only formed a dimer), the starting distance between peptides was $7 \AA$. These (and the 2 nao systems) were then solvated using a TIP3P water model with adequately sized rectangular water boxes, $\sim 90 \AA \times 94 \AA \times 87 \AA$ for the system with excess ions (EI) and $\sim 72 \AA \times 99 \AA \times 87 \AA$ for the system without EIs (NEI). The EI system is understood as a $A b_{1-42}$ system with counterions neutralizing the system and some additional $\mathrm{Na}^{+} / \mathrm{Cl}^{-}$ions to achieve the desired ionic concentration, whereas the system without EIs (NEIs) is one with $\mathrm{Ab}_{1-42}$ neutralized only and no extra ions added. We study two simulations with $\mathrm{Na}^{+}$and $\mathrm{Cl}^{-}$ions added to mimic<smiles>[R]NC(=O)C([CH]c1ccc(O)cc1)NC([R])=O</smiles>
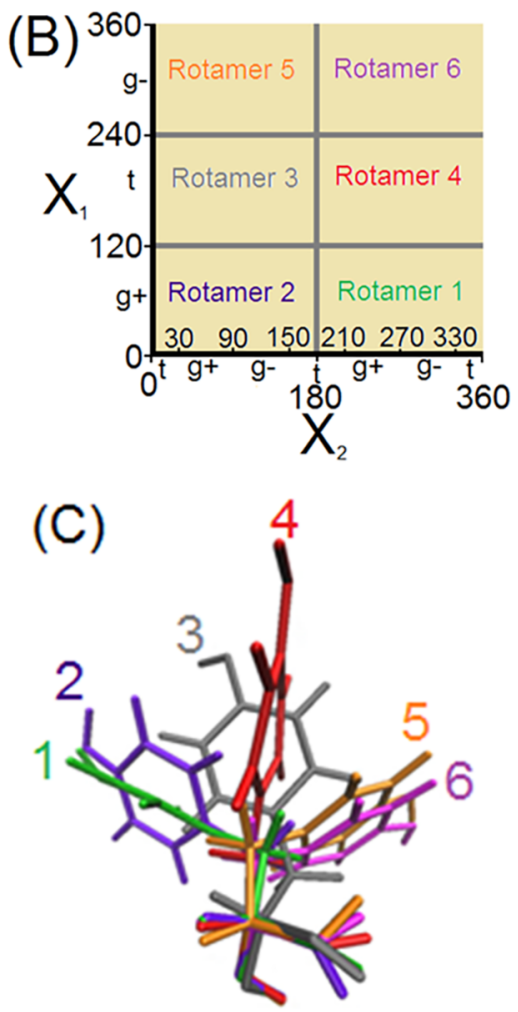

Figure 1. (A) Dihedral angles $\mathrm{C}_{\alpha}-\mathrm{C}_{\beta}$ and $\mathrm{C}_{\beta}-\mathrm{C}_{\gamma}$ used in this work, following ref 28. (B) Dihedral angle quadrants used to identify the rotamer states. $X_{1}$ and $X_{2}$ angle conformations $(\mathrm{g}+, \mathrm{g}-$, and $\mathrm{t}$ ) are indicated. ${ }^{29}$ (C) VMD images of the six rotamer states.

the ionic concentration of blood, $\sim 0.15 \mathrm{~mol} / \mathrm{L}^{27}$ the first is a nonaggregating three monomer system, and the second is a hexamer protofibril.

A water minimization stage (1000 steps) 100 ps of water equilibration was performed on each of these systems. The Langevin piston control pressure was activated with groupbased pressure control, a piston temperature of $300 \mathrm{~K}$, and anisotropic cell fluctuations as in previous work. ${ }^{28} \mathrm{~A}$ minimization phase followed (10000 steps), before a 30 ps period of heating to $300 \mathrm{~K}$ for amorphous systems or $310 \mathrm{~K}$ (body temperature) for the fibril-like systems, both to a pressure of $1 \mathrm{~atm}$. The final thermal equilibration required 270 ps, with a time-step of 1 fs. The production trajectories were performed with a time-step of $2 \mathrm{fs}$, at $300 \mathrm{~K}$ ( or $310 \mathrm{~K}$ ) in the NVT ensemble. The trajectory time varies from 50 to $200 \mathrm{~ns}$ depending on the system studied. Although this is a relatively short simulation time, the early events in the oligomerization process, which are in focus of this work, are captured. The SHAKE algorithm and periodic boundary conditions were employed. van der Waals (vdW) interactions had a cutoff of 12 $\AA$, and PME is used for the electrostatics. 
The tyrosine (Tyr) residue within each $\mathrm{Ab}_{1-42}$ peptide was tracked using VMD to obtain the Tyr rotamer states (preferred orientations). This follows previous work, which showed that the Tyr residues rotate along the $\mathrm{C}_{\alpha}-\mathrm{C}_{\beta}$ and the $\mathrm{C}_{\beta}-\mathrm{C}_{\gamma}$ bonds. ${ }^{28}$ These angles were tracked for each "frame" of the trajectory (every 20000 integration steps) by using the dihedral angles along the sets of atoms $\mathrm{C}-\mathrm{C}_{\alpha}-\mathrm{C}_{\beta}-\mathrm{C}_{\gamma}$ and $\mathrm{C}_{\alpha}-\mathrm{C}_{\beta}-\mathrm{C}_{\gamma}-\mathrm{C}_{\mathrm{D} 1}$, which we label angles $X_{1}$ and $X_{2}$ respectively, as shown in Figure 1A. In this work, each frame of the analyzed trajectory depicts a passage of $0.04 \mathrm{~ns}$; this is chosen to avoid oversampling the $\mathrm{THz}$ molecular vibrations.

At each frame, the dihedral angles are plotted to show the preferred orientations (i.e., rotamer states) of the Tyr residues. We use the range $0^{\circ}-360^{\circ}$ to denote the angles (as opposed to $-180^{\circ}$ to $180^{\circ}$ ) for clarity of presentation. In these plots, we are able to distinguish the states by splitting the $X_{1}-X_{2}$ plane into six zones, as illustrated in Figure 1B. The $X_{2}$ angle is divided into two equal sections, and $X_{1}$ into three. This allows us to quickly and unambiguously identify 6 possible rotamer states (see below for verification that this assignment is consistent with the full set of results obtained in this study). The six rotamer states are illustrated in Figure 1C.

It should be noted that the previous work had only shown the presence of the first four rotamer states, ${ }^{28}$ and the work presented in this paper revealed that the fifth and sixth rotamer states become occupied in the protofibril structures (see Figure $1 \mathrm{C})$. This suggests that they would also appear in larger structures such as amyloid fibrils (see results below). We note that because of the lack of symmetry along the peptide backbone and the unique sequence of the peptide, the rotamer states 5 and 6 are distinct and different to the other states.

\section{RESULTS}

$A b_{1-42}$ Amorphous Aggregation. We aim to characterize the rotamer states, their occupancy, and the transition pathways between them in various small oligomers. We are also interested in how the oligomers form, and whether this influences the rotamer statistics. Therefore, we undertake a series of simulations for the following systems:

- Monomers with EIs and without (NEI);

- Dimers in ionic solutions (EI); and

- A tetramer in an ionic solution (EI).

The aggregation simulations allow the oligomers to form in a natural way, simulating the oligomerization that can occur experimentally as monomers diffuse freely through the solution.

Monomers in Ionic Solution. Figure 2 shows results of simulations with three peptides diffusing in a small water box with an ionic concentration of $0.15 \mathrm{~mol} / \mathrm{L}$ (EI system). The extent of the water box is illustrated by the ions in Figure $2 \mathrm{~A}, \mathrm{~B}$ (the water is not shown for clarity). The dimensions of $A b_{1-42}$ monomers when in a stable folded conformation is $\sim 25 \AA \times$ $24 \AA \times 10 \AA$, and the water box is of size $\sim 83 \AA \times 87 \AA \times 102$ $\AA$. Despite the crowded environment, in this simulation, the monomers do not appear to be interacting with one another. This suggests that aggregation (i.e., the formation of close contacts between peptides) does not occur readily and that perhaps something must initiate it, such as misfolding, ${ }^{30}$ which has been observed in other trajectories below.

The proteins begin close together and yet remain independent with little interaction with one another throughout the trajectory. The changes to the peptides'
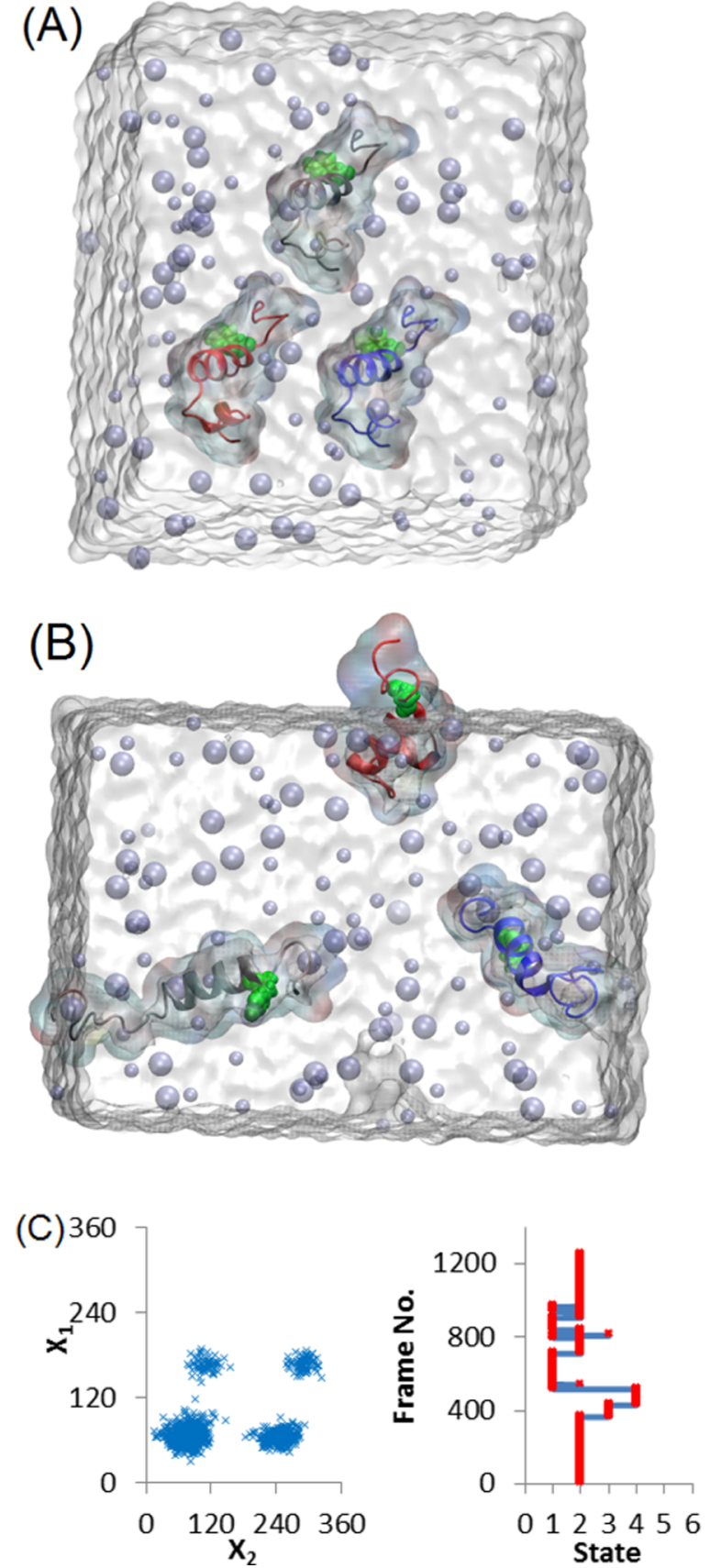

Figure 2. Three-monomer system in an ionic solution (EI): at trajectory time $=0 \mathrm{~ns}$ (A) and after $50 \mathrm{~ns}$ (B) with peptide A shown in blue, peptide $B$ shown in red, and peptide $C$ shown in gray. The $\mathrm{vdW}$ spheres of the ions are shown in gray and Tyr residues are shown in green. In (C), the left-hand graph shows the plots of $X_{1}$ against $X_{2}$ and the right-hand graph shows the rotamer states of peptide A's Tyr residue at each frame, with transitions indicated by the blue lines.

conformations during this trajectory are minimal, as seen in Figure 2B. The heads (low-index residues at the N-terminus) and tails (high index at the C-terminus) of the peptides appear to unravel in a manner similar to that found in the literature, ${ }^{31}$ and the helix sections ${ }^{32,33}$ are focused around residues $11-22$ and 32-36 in all three peptides. This creates the initial " $\mathrm{"}$ " shape of the peptides which might eventually lead to the formation of an antiparallel $\beta$-sheet structure (or hairpin motif). ${ }^{32}$ 

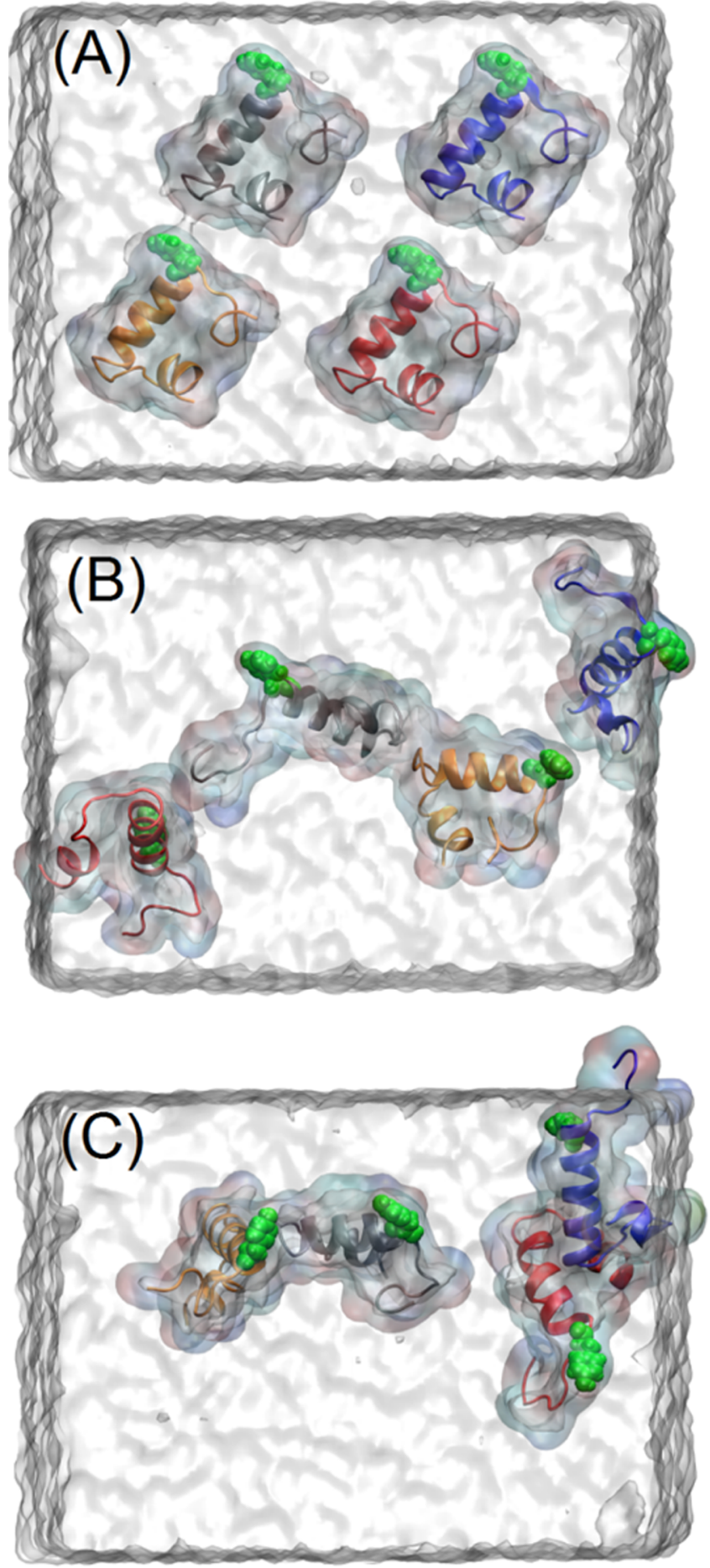

Figure 3. Two-dimer system at time $=0,20$, and 50 ns. Peptide A is shown in blue, peptide $\mathrm{B}$ in red, peptide $\mathrm{C}$ in gray, and peptide $\mathrm{D}$ in orange. The gray vdW spheres for the atoms illustrate the protein surfaces and the Tyr residues are shown in green.

The rotamer states are identified by tracking the two dihedral angles of the Tyr residues, and results for Tyr A are shown in Figure 2C. This clearly shows the rotamer states 1, 2, 3 , and 4 (see Figure 1B) occupied by the Tyr side-chain throughout the trajectory. The rotamers $1-4$ for Tyr A are approximately centered around the $\left(X_{1} ; X_{2}\right)$ angles (in degrees) of $(67 ; 239),(74 ; 81),(166 ; 116)$, and $(168 ; 291)$, respectively. We find similar rotamers for Tyr B at approximately $(66 ; 239)$,
(72;108), (112;164), (166;277), and $(290 ; 170)$, and for Tyr C at approximately $(69 ; 270),(73 ; 67),(168 ; 117)$, and $(166 ; 305)$. A full list of the rotamer centers found in all our simulations is provided in the Supporting Information.

Because the three monomers are relatively unhindered by their surroundings and appear to diffuse freely, their rotamer state occupancies are all similar. As long as they are folded into stable conformations, they appear to show a distinct preference for states 1 and 2 . It can also be noted that there is more frequent movement between states 3 and 4 than in state 2, showing that the Tyr does not find states 3 and 4 favorable for long periods of time in the monomeric peptide. Moreover, Tyr side-chain transitions between the rotamers tend to appear in a cyclic fashion. Transitions observed are $1 \leftrightarrow 2,2 \leftrightarrow 3,3 \leftrightarrow 4$, and $4 \leftrightarrow 1$. This is associated with the proximity of the rotamer states illustrated in Figure $1 \mathrm{C}$.

In order to verify these findings, we have studied many monomer systems for both $A b_{1-42}$ and some $A b_{1-40}$ systems (see the Supporting Information). In the case of $A b_{1-42}$, we have studied two other systems. The first is a three monomer system with no EIs (NEI), and the second (also NEI) contains a monomer and a dimer. These provided a comparison to assess the effect of the screening ions on the Tyr rotamer state occupation and movements.

The results (shown in the Supporting Information) indicate that a visibly more stable conformation is found when the solution ions are present (the EI system). However, we do not attempt to quantify the conformations because all of the amorphous structures are prone to fluctuations as we might expect for small peptides and oligomers. The Tyr side-chains always have a preference toward occupation of states 1 and 2, with a relatively lower population of states 3 and 4, regardless of the presence of the ions. The only exceptions we have observed in the "monomeric" systems were instances of occupation of states 3 and 4 only (or in sometimes only one of these states). However, in these cases, the results can be explained by the close proximity of the Tyr side-chain to surrounding residues or to the backbone of another peptide, preventing free movement between rotamer states, and thus explaining the discrepancy with truly free monomer behavior.

$A b_{1-42}$ Dimers. A $50 \mathrm{~ns}$ trajectory containing four monomers (NEI) that then formed two dimers is illustrated in Figure 3. The water box containing the peptides is $\sim 73 \AA \times$ $99 \AA \times 90 \AA$, which ensures that no cross-boundary interactions occur between a peptide's head and tail because of the periodicity, because the dimensions of the $A b_{1-42}$ monomers (stated previously) are significantly smaller than the total size of the water box.

The initial stages of the trajectory have the four monomers relatively close in proximity. The misfolding or conformational changes shown here are more significant than those seen in the previous monomer systems, which could explain why this system more readily aggregates. ${ }^{26,31}$ Monomers C and D start interacting fairly early and form a dimer by around $20 \mathrm{~ns}$, whereas monomer A moves down, passes through the bottom of the water-box, wraps around to the top of the water-box (due to the periodicity), and begins to interact with monomer $\mathrm{B}$ at approximately $37.5 \mathrm{~ns}$. The last $12.5 \mathrm{~ns}$ of the trajectory contains two dimers, which predominantly have alpha helix structures. ${ }^{32,33}$ The only caveat is that once peptide B begins to aggregate, its alpha helix coil becomes significantly shorter than that in the other peptides for brief periods, changes that have been seen elsewhere. ${ }^{31}$ 
(A)

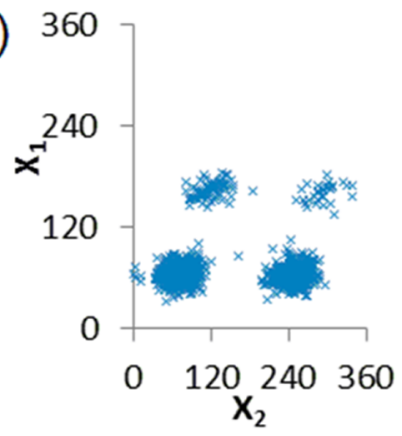

(C)

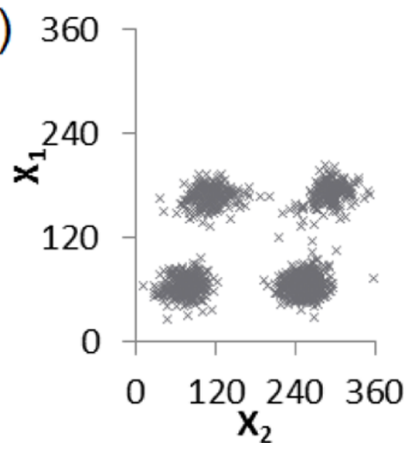

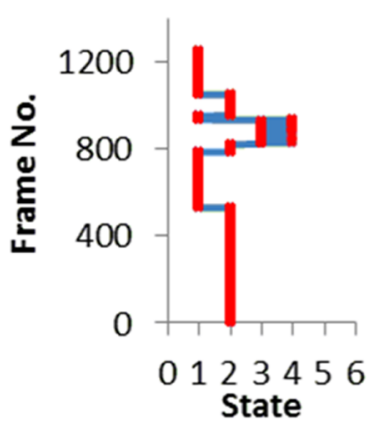

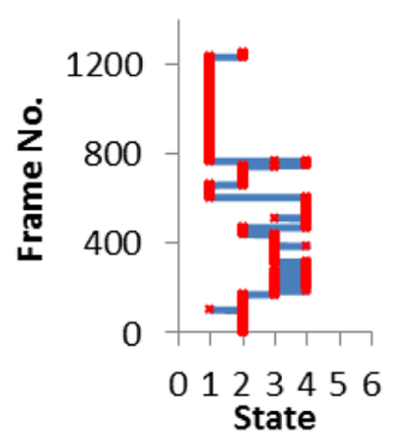

(B)
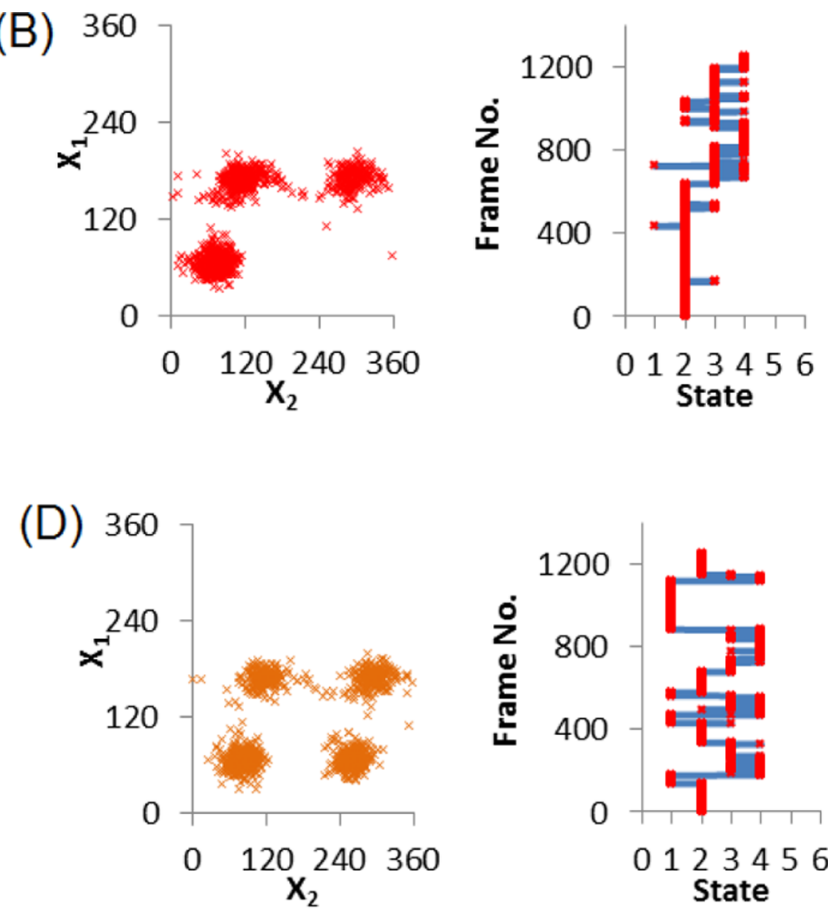

Figure 4. Dimer rotamer states. In each panel, the left graph plots the dihedral angle $X_{1}$ against $X_{2}$, and the right graph shows how the Tyr residues move between the rotamer states. (A-D) Peptides A-D, respectively.

The trends in Figure 4 are repeatedly observed in our $\mathrm{Ab}$ aggregating simulations. Peptides $\mathrm{A}$ and $\mathrm{B}$ spend most of the trajectory as monomers, which is reflected in their Tyr rotamer data. They spend the majority of the early stages in states 1 and 2 , as has been shown to be the preference for monomers. They then show substantial occupation of states 3 and 4 from around frame 800 ( $32 \mathrm{~ns})$, which is the time they begin interacting closely to form a stable aggregate soon after (at $37.5 \mathrm{~ns}$ ). This further suggests that there is an occupational preference of states 1 and 2 when in the monomer form and states 3 and 4 when in an amorphous aggregate because of the more crowded environment.

This pattern is also shown by peptides C and D that aggregate fairly early in the trajectory; there is a rapid shift toward states 3 and 4, with some random fluctuations as might be expected. Tyr C's preference for state 1 near the end of the trajectory suggests that it has become constrained by its surroundings.

$A b_{1-42}$ Tetramer. A short $30 \mathrm{~ns}$ simulation containing six monomers (NEI) is shown in Figure 5. It begins to form an amorphous aggregate early on in the trajectory because of the relatively small water-box surrounding the peptides (dimensions $\sim 72 \AA \times 99 \AA \times 87 \AA$ ), revealing information about the how the Tyr side-chains are affected by their immediate environment.

In Figure 5, the monomers are seen to aggregate within a few nanosecond. This is different from previous simulations, driven by their close proximity which also seems to promote monomer misfolding. ${ }^{32,34}$ The antiparallel $\beta$-sheets that form between the head and tail of peptide $\mathrm{D}$ (folding over on itself to do this) and the head of peptide B (the head creates a small hairpin motif $)^{28}$ are also visible by the end of the trajectory. Other than this all peptides share the features of a helical structure. $^{33}$

Monomers $\mathrm{A}$ and $\mathrm{B}$ begin to aggregate together forming a dimer and monomers $\mathrm{C}$ through $\mathrm{F}$ begin to form a tetramer during the first $4.8 \mathrm{~ns}$. By $7.2 \mathrm{~ns}$, it appears that all six peptides are interacting and show signs of beginning to form an amorphous hexamer. However, at $26.4 \mathrm{~ns}$, peptides $\mathrm{D}$ and $\mathrm{F}$ break away from the aggregate as a dimer and peptides A, B, C, and $\mathrm{E}$ remain as a tetramer for the remainder of the trajectory. We will now focus on the behavior of the Try residues in these four peptides that make this tetramer.

Despite the short duration of this simulation, we obtain informative data about the rotamer states at the early stages of the oligomerization process, as shown in Figure 6. Tyr A is mostly unaffected by its surroundings and shows a distinct preference to states 1 and 2 similar to the behavior of some monomers. Tyr B spends much of the trajectory with freedom to move, but does interact with the aggregate at $\sim 20 \mathrm{~ns}$, which is reflected in a short-lived occupation of states 3 and 4 at around 20 ns (frame 500 in Figure 6), which further suggests that states 3 and 4 are occupied when local interactions affect (but do not trap) the Tyr side-chain. Tyr C has similar results with some occupation of states 3 and 4 most likely associated with closeness of the neighboring residues and surrounding aggregate. Tyr E shows a preference to states 3 and 4 influenced by the close interactions of its neighboring residues and perhaps also the proximity to peptide C.

From this, it is notable that once aggregated, these Tyr residues have a tendency to prefer states 3 and 4, behavior observed above for the dimers. However, this phenomenon is not universally true because of the random nature of the amorphous aggregation process that determines the immediate environment of the Tyr residues.

$A b_{1-42}$ Protofibril Systems. We now aim to contrast the rotamer state statistics of the amorphous oligomers to those observed in a model protofibril structure. Specifically, we characterize the rotamer behavior in the following systems:

- Protofibril hexamer without EIs (NEI); and

- Protofibril hexamer with EIs. 

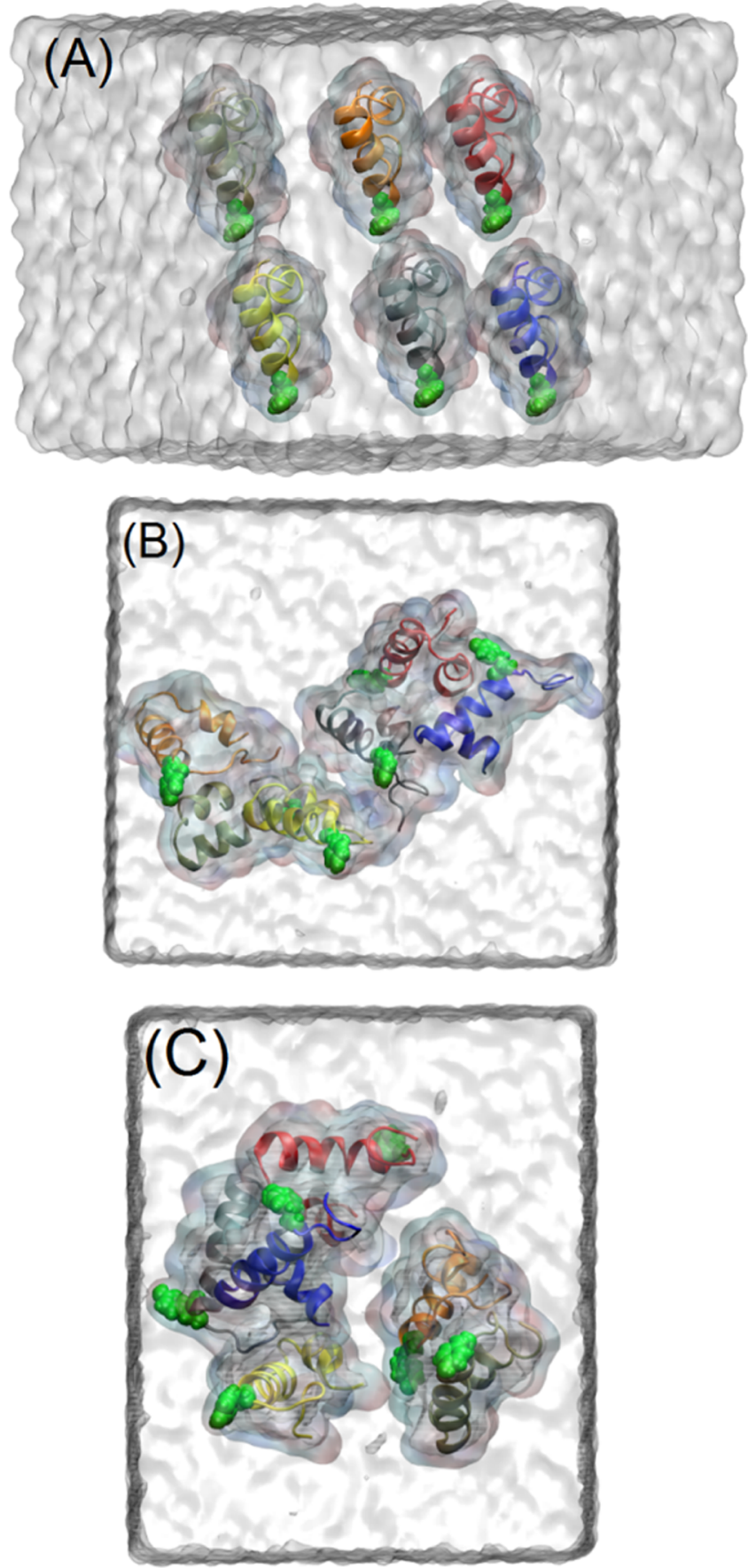

Figure 5. Six-monomer system aggregating to an amorphous tetramer and a dimer, at times 0 (A), 20 (B), and $30 \mathrm{~ns}$ (C). Peptides A-F are shown in blue, red, gray, orange, yellow, and tan, respectively. Tyr residues are shown in green.

Hexamer without Els in Solution (NEI). The final type of system discussed in this paper (in comparison with the monomer and amorphous aggregate systems) is the protofibril hexamer structure (both EI and NEI systems) shown in Figure 7 which has been created from 2nao.pdb. ${ }^{26}$ Here, the individual peptides have unravelled, losing their helical structure, and have formed strong $\beta$-sheet bonds. It has been proposed that this is the basic building block required in the formation of fibrils. The peptides in this hexamer have a very different structure to those in the amorphous aggregates discussed above, and as we will show, this causes changes to the Tyr rotamer statistics.

In the simulated structure, the hexamer has dimensions $\sim 84$ $\AA \times 52 \AA \times 14 \AA$ and the overall water-box size is large enough $(\sim 90 \AA \times 94 \AA \times 87 \AA)$ to ensure that there is no aggregation artificially induced by the periodic boundaries, although there could be some long-range interactions affecting the Tyr sidechain movements. A larger water-box would be beneficial, but the increased size of the calculation would increase the computational costs.

This trajectory starts with the protofibril in a configuration found from NMR experimentation (see Figure 7A) and therefore should be stable. However, as the NEI trajectory begins, the backbone regions around the Tyr side-chains of peptides $\mathrm{D}, \mathrm{E}$, and $\mathrm{F}$ spread apart, whereas $\mathrm{A}, \mathrm{B}$, and $\mathrm{C}$ remain rigid and relatively close together. After $20 \mathrm{~ns}$, there are some significant conformational changes visible near the head of the peptides in the stack containing peptides A-C. In contrast, the changes seen for peptides D-F are less significant. By $40 \mathrm{~ns}$, the structure has moved further from the original conformational shape, with a lot of the $\beta$-sheet sections beginning to shift, but it can still be identified as a hexamer protofibril. After $60 \mathrm{~ns}$, the stacked portion containing peptides $\mathrm{A}-\mathrm{C}$ is further misshapen but its $\beta$-sheet structuring has mostly remained intact and the stack containing $\mathrm{D}-\mathrm{F}$ remains relatively structured throughout. After $100 \mathrm{~ns}$, the aggregate maintains the structure it has at that point for approximately $30 \mathrm{~ns}$ and then the middle sections of the peptides' backbones spread out again.

As apparent in Figure 7B, by the end of the NEI simulation, the backbones of the peptides are not aggregated as rigidly as might be expected and they are further apart in the middle of the structure than they were at the start of the trajectory. It should also be noted that Tyr E (in the middle layer of one side of the hexamer stack) has significantly more room to move than the equivalent Tyr B in the other side of the hexamer.

The final structure of the system is as follows: peptides A, B, and $\mathrm{C}$ have parallel $\beta$-sheets formed between Ile $31-$ Val36 and Val39-Val40. Peptides A and B also have a $\beta$-sheet between Glu3-Arg5, which was lost in C. Furthermore, the initial $\beta$ sheets in the mid-section of these peptides have disappeared, which is why the mid-sections spread apart as discussed above. Peptides D, E, and $\mathrm{F}$ remain structured throughout, with $\beta$ sheets around residues Phe4-Hsd6, Gln15-Phe20, and Ile31-Val36; it has been seen in other trajectories which only contained, for example, peptides $\mathrm{A}-\mathrm{C}$ structured in a manner similar to those seen in this hexamer. $\mathrm{E}$ and $\mathrm{F}$ also contain a $\beta$ sheet at the tail around Val39-Val40. The lack of stability might be caused by the low ion count in the system, so that long-range screening effects are missing. This becomes more apparent when we compare to the same system simulated in an ionic solution (EI) as discussed below.

The rotamer states for peptides A, C, E, and F from the EI simulation are shown in Figure 8. For these fibril structures, there is a more significant shift toward rotamer states 3 and 4 with respect to the amorphous aggregates, as well as the occupation of states 5 and 6 . There are exceptions however; Tyr D's environment is more akin to that of a monomer than an aggregate, and similarly Tyr E's side-chain moves quite freely and spends about $25 \%$ of its time in states 1 and 2 (see Figure $8 \mathrm{C}$ ). These variations are expected because of the stochastic nature of the simulations. 

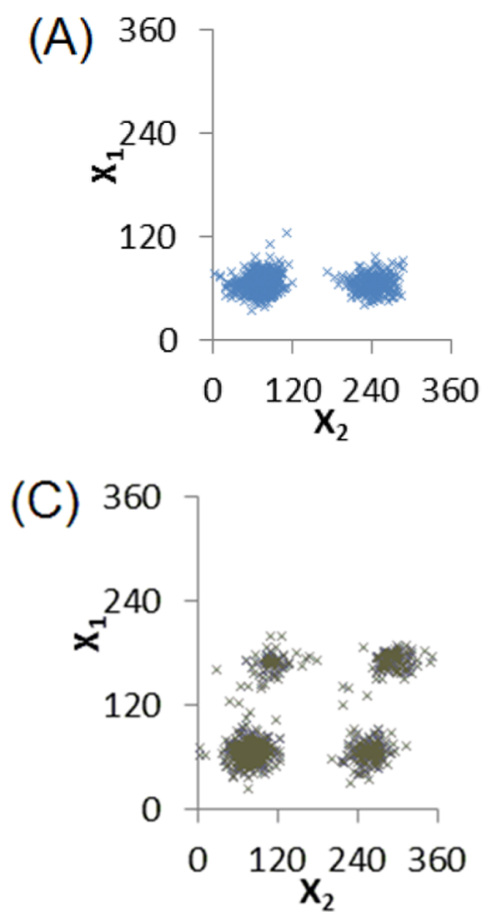
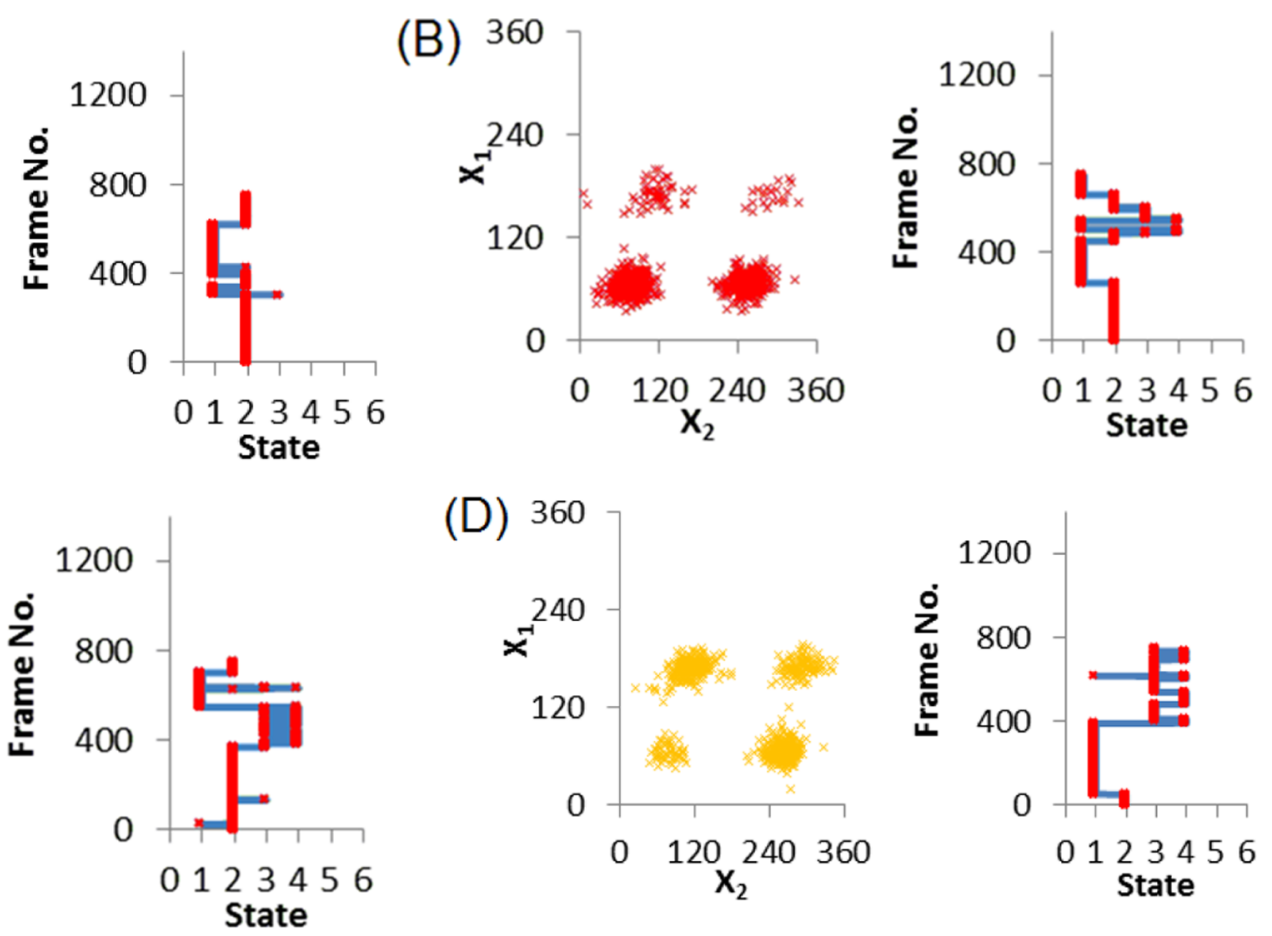

Figure 6. Tetramer rotamer states. In each panel, the left graph plots the dihedral angle $X_{1}$ against $X_{2}$, and the right graph shows how the Tyr residues move between the rotamer states. (A-D) Peptides A, B, C, and E, respectively.

Nevertheless, there does seem to be a more distinct feature arising in the rotamer statistics. The most notable difference between this protofibril and the previous simulations is the appearance of rotamer states 5 and 6 in peptides A, C, E, and F. Despite not being seen previously, it is evident that these are distinct states that can be occupied at different points throughout the trajectory, with finite probabilities of occurring.

As there is a recurring pattern of the Tyr side-chains moving between states in an ordered fashion (from $1 \leftrightarrow 2,2 \leftrightarrow 3,3 \leftrightarrow$ 4 , and so on), it is reasonable to suppose that the sudden appearance of states 5 and 6 could be enabled by a Tyr sidechain spending more time in states 3 and 4, causing it to have a higher probability of transitioning from state 4 into state 5 or 6 . Another likely reason for the appearance of these new states may be the close proximity of the surrounding residues forcing the Tyr side-chain to flex and rotate more aggressively, allowing it to enter otherwise unfavorable states 5 and 6 .

Hexamer in Ionic Solution (El). As can be seen above, the hexamer structure shown in Figure $7 \mathrm{C}$ is similar to that of Figure $7 \mathrm{~B}$. The inclusion of an ionic concentration of 0.25 $\mathrm{mol} / \mathrm{L} \mathrm{(EI)} \mathrm{is} \mathrm{the} \mathrm{only} \mathrm{difference} \mathrm{in} \mathrm{the} \mathrm{simulations;}$ nevertheless, visual inspection of the trajectory indicates that long-range screening effects ${ }^{35}$ might lead to the development of more stable form of the oligomer. The water-box size is similar to that used previously $(\sim 92 \AA \times 95 \AA \times 90 \AA)$ which is big enough for the hexamer. This trajectory was also performed for $200 \mathrm{~ns}$, but as there was no real change in structure after the first $110 \mathrm{~ns}$, we show the structure at this time in Figure $7 \mathrm{C}$.

The trajectory begins in the same structure as above (Figure 7A). The structure remains relatively unchanged for the first 10 ns, but with some minor twisting near the head of the stack containing peptides A-C. After $40 \mathrm{~ns}$, the overall hexamer structure remains relatively unchanged, except some noticeable twisting of the mid-section of both stacks. Nothing of interest occurs until 70 ns where Tyr A and D appear to gain some freedom and move around more freely, though the aggregate itself remains significantly more stable throughout the $200 \mathrm{~ns}$ trajectory than all previous aggregates. Throughout the trajectory, the $\beta$-sheet sections holding the peptides together shift and move, but the aggregate is significantly more stable than seen above. Peptides A and B have $\beta$-sheet sections within the regions of Glu3-Arg5, Gln15-Phe20, Ile31-Gly33, and Gly38-Ile41. Peptide $\mathrm{C}$ has $\beta$-sheet sections in similar positions, however as it is not as tightly aggregated it has some variation (Gln15-Leu17 rather than Gln15-Phe20). Peptides D and E have $\beta$-sheet regions at Glu3-Arg5, Lys16Val18, Ala30-Val36, and Val39-Ile41. Peptide F also has them in these locations, except at the head (Glu3-Arg5) which has separated itself from the other two peptides.

The rotamer states for Tyr A, B, C, and F in this trajectory show an overwhelming preferences toward states 3 and 4, but do not enter states 5 or 6 at all. Tyr D not only shows a distinct preference to states 3 and 4 but also temporarily enters states 5 and 6, as shown in Figure 8E. Tyr E (shown in Figure 8F) also enters states 5 and 6 briefly at the start of the trajectory.

\section{ROTAMER STATE SUMMARY}

Figure 9 presents the angles obtained for the six rotamers identified in the simulations above; further details are given in Supporting Information Table S1. Rotamer 1 is centered (has mean coordinates $)$ at $\left(X_{1} ; X_{2}\right)=(71 ; 265)$ which, according to the definition introduced in Figure 1 , is equivalent to $(\mathrm{g}+; \mathrm{g}+)$; rotamer 2 is centered at $\left(X_{1} ; X_{2}\right)=(72 ; 76)=(\mathrm{g}+; \mathrm{g}+)$; rotamer 3 at $\left(X_{1} ; X_{2}\right)=(165 ; 92)=(\mathrm{t} ; \mathrm{g}-)$; rotamer 4 at $\left(X_{1} ; X_{2}\right)=$ $(166 ; 270)=(\mathrm{t} ; \mathrm{g}+/ \mathrm{g}-)$; rotamer 5 at $\left(X_{1} ; X_{2}\right)=(285 ; 80)=$ $(\mathrm{g}-; \mathrm{g}+)$; and rotamer 6 at $\left(X_{1} ; X_{2}\right)=(285 ; 265)=(\mathrm{g}-; \mathrm{g}+)$. The range of the angular variation for each rotamer depends on the immediate environment of the Tyr side-chain. Despite the outliers caused by statistical fluctuations, there is 

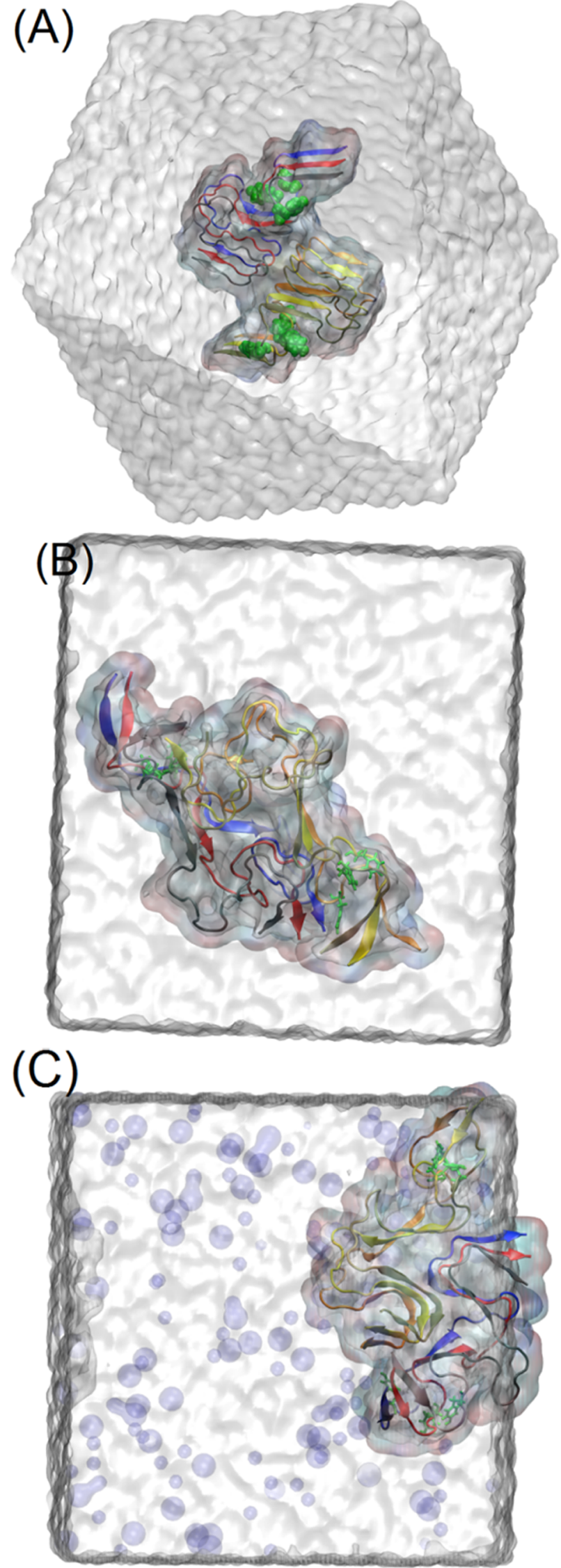

Figure 7. (A) Initial configuration of a stable protofibril hexamer, with water shown to indicate the water-box size. (B) $\beta$-sheet hexamer without screening ions (NEI system) depicted at time 200 ns. (C) $\beta$ -
Figure 7. continued

sheet hexamer in $0.25 \mathrm{~mol} / \mathrm{L}$ ionic solution (EI system) depicted at time 110 ns. Peptides A-F are shown in blue, red, gray, orange, yellow, and tan, respectively. The ions are shown as gray vdW spheres and the Tyr residues are shown in green.

consistency in the general areas occupied by the rotamers, regardless of aggregate type (monomer, amorphous, or protofibrillar). In a recent quantum chemical study, only four Tyr rotamers (a combination of $\mathrm{g}+$ and $\mathrm{g}-$ ) were identified. ${ }^{36}$ The discrepancy probably comes from the fact that in quantum calculations, only single molecules were taken into account and environmental effects could not be fully included for the Tyr side-chain.

As the amorphous aggregate becomes more tightly packed, the Tyr side-chains will interact more closely with surrounding residues/backbones of other peptides and will have less exposure to water. This affects the Tyr side-chain movements, and they begin to more readily flex into positions that are unfavorable in a monomer.

As is indicated in Table 1, monomers show some occupation of the first 4 rotamer states but with preference to states 1 and 2 as described previously. ${ }^{28}$ The amorphous aggregates show approximately equal preference for all states 1-4; however, this could be misleading; the peptides begin as monomers before eventually stabilizing as an aggregate, which means that the occupancy of states 1 and 2 could still be biased by the initial stages of aggregation, although it is difficult to precisely distinguish this effect in the simulation data.

\section{CONCLUSIONS}

The series of simulations reported here show that the rotamer state occupation of the Tyr side-chains is strongly dependent on the Tyr side-chain's immediate environment. Consequently, amorphous aggregates have a different rotamer profile to monomers, and the protofibril $\beta$-sheet structures reveal two new rotamer states that appear to be uniquely associated with them. This observation could pave the way to use intrinsic fluorescence of the Tyr residues to monitor not only the initial, amorphous stages of $\mathrm{Ab}$ aggregation (as done previously ${ }^{28}$ ), but also to detect the onset of fibril formation without using the extrinsic fluorescence sensors indicating appearance of $\beta$ sheets or fibrillisation (e.g., ThioflavinT) that might interfere with the natural aggregation. Indeed, the existence of six different Tyr rotamers in the heterogenic Ab oligomers may be confirmed if each rotamer has its distinctive fluorescence characteristics (e.g., fluorescence lifetime). In this case, the contributions of the Tyr rotamers in the aggregating $\mathrm{Ab}$ could be determined in fluorescence intensity decay measurements, providing that the quantum yield of all rotamers is sufficiently high to be identified in the fluorescence signal.

Our results also suggest that excess solution ions may cause protein conformational changes because of the electrostatic screening effect. This could affect the stabilization or conformation of an aggregate and potentially make more tightly (stable) aggregated systems which may allow for rotamers 5 and 6 to appear more readily in protofibrils. The ionicity-induced changes in the Tyr surroundings, together with the direct ion-Tyr interactions, are likely to modify the experimentally observed fluorescence responses.

The rotamers 5 and 6 (illustrated in Figure 1C) are a novel finding from this work. These states do not appear to be 

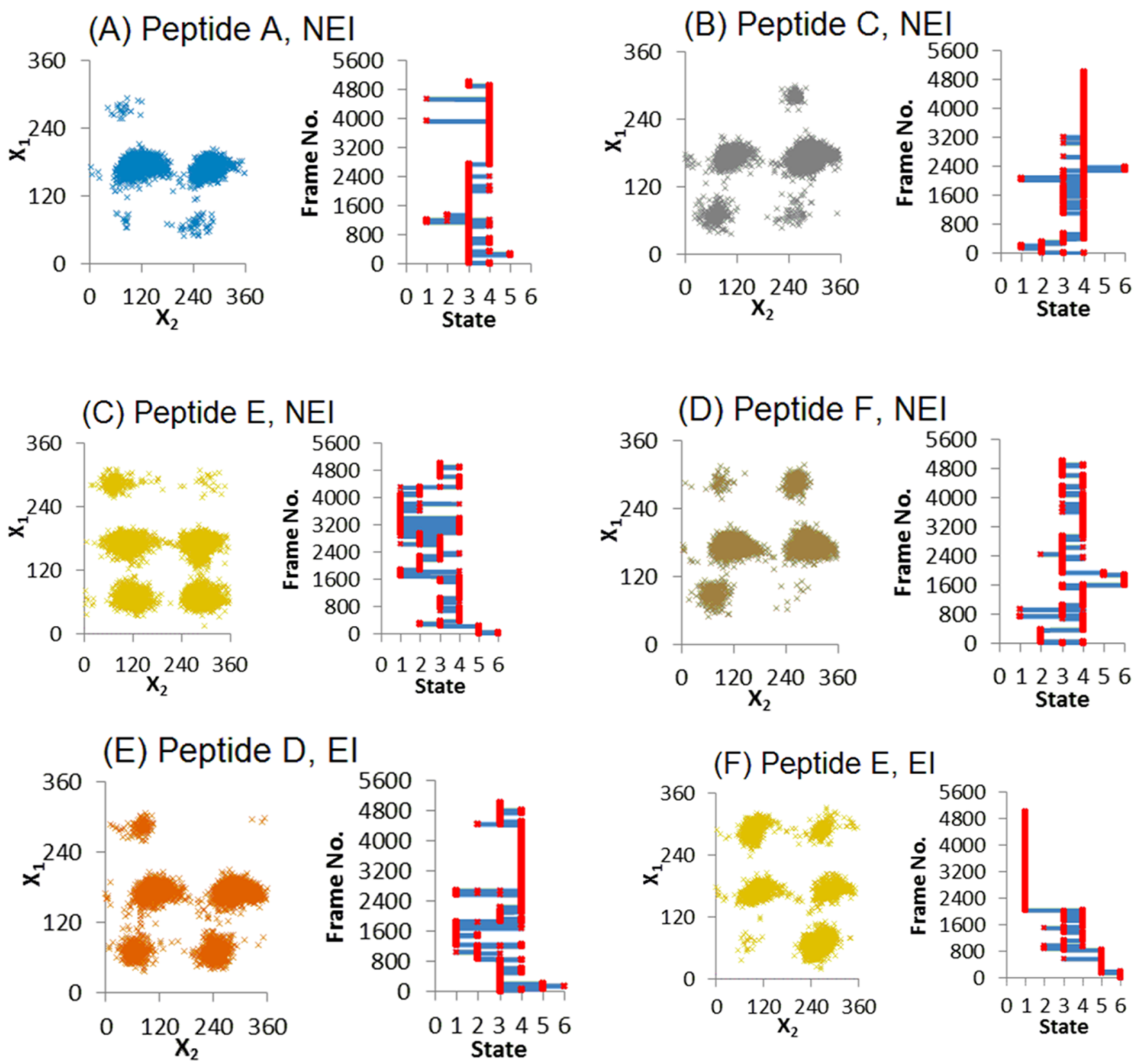

Figure 8. Sample results of protofibril hexamer rotamers with (EI) and without ions (NEI) in solution. In each panel, the left graphs show the dihedral angle $X_{1}$ against the dihedral angle $X_{2}$, and the right graphs show how the Tyr residues move between the rotamer states. (A-C) Peptides A-C, respectively, in NEI systems, whereas (D-F) show peptides F, D, and E, respectively, in EI systems.

artefacts as they are present at various stages of the different protofibril trajectories. However, we note that they do not occur in the second half of our trajectories, and we believe that this might be due to the instability of our small protofibril fragments arising from their short length, a feature that would be mitigated in larger systems with longer protofibrils; further work on this point is required. It is also clear from the results that these states are directly caused by the stacking mechanism, rather than the mirrored protein within a single layer of a fibril. The detection of these new rotamers may still be possible despite their low occupation (see Table 1). This is because although they only appear occasionally in our quasi-stable structures, in larger fibrils they will appear more often as there are more Tyr residues that will also be more tightly packed together. Experimental work using fluorescence spectroscopy could potentially be used to help identify these new rotamer states through lifetime analysis in non-aggregated and aggregating systems. Detection of these states could provide better understanding of the aggregation and fibrillation kinetics and also provide a label-free method to study the influence of additives on the aggregation, paving the way to enhanced drug discovery studies.

\section{ASSOCIATED CONTENT}

S Supporting Information

The Supporting Information is available free of charge on the ACS Publications website at DOI: 10.1021/acsomega.8b02408.

We provide full sets of rotamer statistics from the simulations using three $\mathrm{Ab}_{1-42}$; a set from a $\mathrm{Ab}_{1-40}$ 


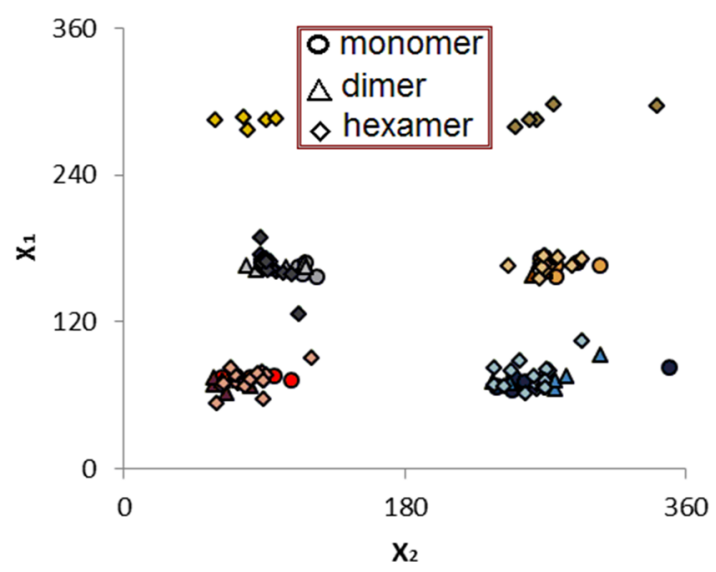

Figure 9. $\left(X_{1} ; X_{2}\right)$ angles of Tyr associated with each peptide in the various systems studied. Rotamers $1,2,3,4,5$, and 6 are shown as blue, red, gray, orange, yellow, and tan shade centers, respectively.

Table 1. Proportion of Time Spent in the Various Rotamer States in Different Simulated Structures ${ }^{a}$

\begin{tabular}{|cccc} 
& $\begin{array}{c}\mathrm{Ab}_{1-42} \\
\text { monomer }\end{array}$ & $\begin{array}{c}\mathrm{Ab}_{1-42} \text { amorphous } \\
\text { aggregate }\end{array}$ & $\begin{array}{c}\mathrm{Ab}_{1-42} \\
\text { protofibril }\end{array}$ \\
\hline state 1 & 0.375 & 0.256 & 0.176 \\
state 2 & 0.438 & 0.232 & 0.176 \\
state 3 & 0.125 & 0.256 & 0.297 \\
state 4 & 0.063 & 0.256 & 0.275 \\
state 5 & & & 0.044 \\
state 6 & & & 0.033
\end{tabular}

${ }^{a}$ The error is below 0.001 because of the large number of frames from the set of simulations presented above.

dimer; the sets from the six $A b_{1-42}$ simulation and from the protofibril simulations for EI and NEI systems; and we also tabulate the central coordinates of the rotamer states found in our various $A b_{1-42}$ and $A b_{1-40}$ simulations (PDF)

\section{AUTHOR INFORMATION}

\section{Corresponding Authors}

*E-mail: karina.kubiak@strath.ac.uk (K.K.-O.).

*E-mail: paul.mulheran@strath.ac.uk (P.A.M.).

\section{ORCID $\odot$}

Karina Kubiak-Ossowska: 0000-0002-2357-2111

\section{Notes}

The authors declare no competing financial interest.

The data-set allowing for the reproduction of the trajectories as well as sample trajectories analyzed in the paper are deposited under DOI: http://dx.doi.org/10.15129/ef1209ba-3869-40a38603-4381d4dc0138.

\section{ACKNOWLEDGMENTS}

The MD simulations were performed on the EPSRC funded ARCHIE-WeSt High Performance Computer (www.archiewest.ac.uk); EPSRC grant no. EP/K000586/1. OM was supported by a University of Strathclyde studentship.

\section{REFERENCES}

(1) Brodney, M. A.; Lau, L. Alzheimer's Disease (Topics in Medicinal Chemistry); Springer, 2010.
(2) Guerchet, M.; Prina, M.; Prince, M. World Alzheimer Report 2013: Journey of Caring: An analysis of long-term care for dementia; King's College London, 2013.

(3) Stroud, J. C.; Liu, C.; Teng, P. K.; Eisenberg, D. Toxic fibrillar oligomers of amyloid- have cross- structure. Proc. Natl. Acad. Sci. U.S.A. 2012, 109, 7717-7722.

(4) Bedrood, S.; Li, Y.; Isas, J. M.; Hegde, B. G.; Baxa, U.; Haworth, I. S.; Langen, R. Fibril Structure of Human Islet Amyloid Polypeptide. J. Biol. Chem. 2012, 287, 5235-5241.

(5) Benveniste, O.; Stenzel, W.; Hilton-Jones, D.; Sandri, M.; Boyer, O.; van Engelen, B. G. M. Amyloid Deposits and Inflammatory Infiltrates in Sporadic Inclusion Body Myositis: the Inflammatory Egg Comes Before the Degenerative Chicken. Acta Neuropathol. 2015, 129, 611-624.

(6) Hiltunen, M.; van Groen, T.; Jolkkonen, J. Functional Roles of Amyloid- $\beta$ Protein Precursor and Amyloid- $\beta$ Peptides: Evidence from Experimental Studies. J. Alzheimer's Dis. 2009, 18, 401-412.

(7) Sadigh-Eteghad, S.; Talebi, M.; Farhoudi, M.; Golzari, S. E. J.; Sabermarouf, B.; Mahmoudi, J. Beta-Amyloid Exhibits Antagonistic Effects on Alpha 7 Nicotinic Acetylcholine Receptors in Orchestrated Manner. J. Med. Hypotheses Ideas 2014, 8, 49-52.

(8) Luo, Y.; Bolon, B.; Damore, M. A.; Fitzpatrick, D.; Liu, H.; Zhang, J.; Yan, Q.; Vassar, R.; Citron, M. BACE1 ( $\beta$-secretase) knockout mice do not acquire compensatory gene expression changes or develop neural lesions over time. Neurobiol. Dis. 2003, 14, 81-88.

(9) Bogoyevitch, M. A.; Boehm, I.; Oakley, A.; Ketterman, A. J.; Barr, R. K. Targeting the JNK MAPK Cascade for Inhibition: Basic Science and Therapeutic Potential. Biochim. Biophys. Acta 2004, 1697, 89-101.

(10) Tabaton, M.; Zhu, X.; Perry, G.; Smith, M. A.; Giliberto, L. Signaling effect of amyloid- $\beta 42$ on the processing of A $\beta$ PP. Exp. Neurol. 2010, 221, 18-25.

(11) Zuo, K.; Gong, J.-S.; Yanagisawa, K.; Michikawa, M. A Novel Function of Monomeric Amyloid Beta-Protein Serving as an Antioxidant Molecule Against Metal-Induced Oxidative Damage. J. Neurosci. 2002, 22, 4833-4841.

(12) Baruch-Suchodolsky, R.; Fischer, B. A $\beta 40$, either Soluble or Aggregated, Is a Remarkably Potent Antioxidant in Cell-Free Oxidative Systems. Biochemistry 2009, 48, 4354-4370.

(13) Yao, Z.-X.; Papadopoulos, V. Function of $\beta$-amyloid in cholesterol transport: a lead to neurotoxicity. FASEB J. 2002, 16, 1677-1679.

(14) Igbavboa, U.; Sun, G. Y.; Weisman, G. A.; He, Y.; Wood, W. G. Amyloid $\beta$-protein stimulates trafficking of cholesterol and caveolin-1 from the plasma membrane to the Golgi complex in mouse primary astrocytes. Neuroscience 2009, 162, 328-338.

(15) Soscia, S. J.; Kirby, J. E.; Washicosky, K. J.; Tucker, S. M.; Ingelsson, M.; Hyman, B.; Burton, M. A.; Goldstein, L. E.; Duong, S.; Tanzi, R. E.; Moir, R. D. The Alzheimer's Disease-Associated Amyloid $\beta$-Protein Is an Antimicrobial Peptide. PLoS One 2010, 5, e9505.

(16) Maloney, B.; Lahiri, D. K. The Alzheimer's amyloid $\beta$-peptide $(\mathrm{A} \beta)$ binds a specific DNA $\mathrm{A} \beta$-interacting domain $(\mathrm{A} \beta \mathrm{ID})$ in the $\mathrm{APP}, \mathrm{BACE} 1$, and APOE promoters in a sequence-specific manner: Characterizing a new regulatory motif. Gene 2011, 488, 1-12.

(17) Bailey, J. A.; Maloney, B.; Ge, Y.-W.; Lahiri, D. K. Functional activity of the novel Alzheimer's amyloid $\beta$-peptide interacting domain $(\mathrm{A} \beta \mathrm{ID})$ in the APP and BACE1 promoter sequences and implications in activating apoptotic genes and in amyloidogenesis. Gene 2011, 488, 13-22.

(18) Marsden, I. T.; Minamide, L. S.; Bamburg, J. R. Amyloid- $\beta$ Induced Amyloid- $\beta$ Secretion: A Possible Feed-Forward Mechanism in Alzheimer's Disease. J. Alzheimer's Dis. 2011, 24, 681-691.

(19) Lindgren, M.; Sörgjerd, K.; Hammarström, P. Detection and Characterization of Aggregates, Prefibrillar Amyloidogenic Oligomers, and Protofibrils Using Fluorescence Spectroscopy. Biophys. J. 2005, $88,4200-4212$.

(20) Herzig, H. P.; Neier, R.; Santi, S.; Barakat, E. Bloch Meets Alzheimer. SPG Mitteilungen 2014, 42, 11-14. 
(21) Khurana, R.; Coleman, C.; Ionescu-Zanetti, C.; Carter, S. A.; Krishna, V.; Grover, R. K.; Roy, R.; Singh, S. Mechanism of Thioflavin T Binding to Amyloid Fibrils. J. Struct. Biol. 2005, 151, 229-238.

(22) Mancini, O.; Wellbrock, T.; Rolinski, O. J.; Kubiak-Ossowska, K.; Mulheran, P. A. Probing Beta Amyloid Aggregation Using Fluorescence Anisotropy: Experiments and Simulation. Phys. Chem. Chem. Phys. 2018, 20, 4216-4225.

(23) Phillips, J. C.; Braun, R.; Wang, W.; Gumbart, J.; Tajkhorshid, E.; Villa, E.; Chipot, C.; Skeel, R. D.; Kalé, L.; Schulten, K. Scalable Molecular Dynamics with NAMD. J. Comput. Chem. 2005, 26, 17811802.

(24) Humphrey, W.; Dalke, A.; Schulten, K. VMD: Visual Molecular Dynamics. J. Mol. Graphics 1996, 14, 33-38.

(25) Crescenzi, O.; Tomaselli, S.; Guerrini, R.; Salvadori, S.; D’Ursi, A. M.; Temussi, P. A.; Picone, D. Solution structure of the Alzheimer amyloid $\beta$-peptide (1-42) in an apolar microenvironment. Eur. J. Biochem. 2002, 269, 5642-5648.

(26) Wälti, M. A.; Ravotti, F.; Arai, H.; Glabe, C. G.; Wall, J. S.; Böckmann, A.; Güntert, P.; Meier, B. H.; Riek, R. Atomic-resolution structure of a disease-relevant $\mathrm{A} \beta(1-42)$ amyloid fibril. Proc. Natl. Acad. Sci. U.S.A. 2016, 113, E4976-E4984.

(27) Butt, H.; Graf, K.; Kappl, M. Poison Boltzmann Theory of the Diffuse Double Layer, Physics and Chemistry of Interfaces; Wiley-VCH: Berlin, 2003; p 45.

(28) Amaro, M.; Kubiak-Ossowska, K.; Birch, D. J. S.; Rolinski, O. J. Initial stages of beta-amyloid $\mathrm{A} \beta 1-40$ and $\mathrm{A} \beta 1-42$ oligomerization observed using fluorescence decay and molecular dynamics analyses of tyrosine. Methods Appl. Fluoresc. 2013, 1, 015006.

(29) www.dynameomics.org/rotamer/Tyr.aspx.

(30) Ashraf, G.; Greig, N.; Khan, T.; Hassan, I.; Tabrez, S.; Shakil, S.; Sheikh, I.; Zaidi, S.; Akram, M.; Jabir, N.; Firoz, C.; Naeem, A.; Alhazza, I.; Damanhouri, G.; Kamal, M. Protein Misfolding and Aggregation in Alzheimer's Disease and Type 2 Diabetes Mellitus. CNS Neurol. Disord. -Drug Targets 2014, 13, 1280-1293.

(31) Vivekanandan, S.; Brender, J. R.; Lee, S. Y.; Ramamoorthy, A. A partially folded structure of amyloid-beta(1-40) in an aqueous environment. Biochem. Biophys. Res. Commun. 2011, 411, 312-316.

(32) Chauhan, A.; Varma, A. A Textbook of Molecular Biotechnology; I.K. International Publishing House Pvt Ltd.: New Delhi, 2009; p 32. Crevenna, A. H.; Naredi-Rainer, N.; Lamb, D. C.; Wedlich-Söldner, R.; Dzubiella, J. Effects of Hofmeister Ions on the $\alpha$-Helical Structure of Proteins. Biophys. J. 2012, 102, 907-915.

(33) Crevenna, A. H.; Naredi-Rainer, N.; Lamb, D. C.; WedlichSöldner, R.; Dzubiella, J. Effects of Hofmeister Ions on the $\alpha$-Helical Structure of Proteins. Biophys. J. 2012, 102, 907-915.

(34) Hortschansky, P.; Schroeckh, V.; Christopeit, T.; Zandomeneghi, G.; Fändrich, M. The aggregation kinetics of Alzheimer's $\beta$-amyloid peptide is controlled by stochastic nucleation. Protein Sci. 2005, 14, 1753-1759.

(35) Gebbie, M. A.; Dobbs, H. A.; Valtiner, M.; Israelachvili, J. N. Long-Range Electrostatic Screening in Ionic Liquids. Proc. Natl. Acad. Sci. U.S.A. 2015, 112, 7432-7437.

(36) Yang, B.; Liu, S.; Lin, Z. Computational study on single molecular spectroscopy of tyrosinglycine, tryptophane-glycine and glycine-tryptophane. Sci. Rep. 2017, 7, 15869. 